\title{
Resource competition and coexistence in heterogeneous metacommunities: Many-species coexistence is unlikely to be facilitated by spatial variation in resources
}

There is little debate of about the potential of environmental heterogeneity to facilitate species diversity. However, attempts to show the relationship between spatial heterogeneity and diversity empirically have given mixed results. One reason for this may be failure to consider how species respond to the factor in the environment that varies. Most models of the heterogeneity-diversity relationship assume heterogeneity in non-resource environmental factors. These models show the potential for spatial heterogeneity to promote many-species coexistence via mainly the spatial storage effect. Here, I present a model of species competition under spatial heterogeneity a resource factor. This model allows for the stable coexistence of only two species. Partitioning the model to quantify the contributions of variation-dependent coexistence mechanisms shows contributions from only one mechanism, growth-density covariance. More notably, it shows the lack of potential for any contribution from the spatial storage effect, the only mechanism that can facilitate stable many-species coexistence. This happens because the spatial storage effect measures the contribution of different species to specializing on different parts of the gradient of the heterogeneous factor. Under simple models of resource competition, in which all species grow best at high resource levels, such specialization is impossible. This analysis suggests that, in the absence of additional mechanisms, spatial heterogeneity in a single resource is unlikely to facilitate many-species coexistence and, more generally, that when evaluating the relationship between heterogeneity and diversity, a distinction should be made between resource and non-resource factors. 
2 Donald R. Schoolmaster Jr.

3choolm4@msu.edu

4 W.K. Kellogg Biological Station

5 Michigan State University

$6 \quad 3700$ E. Gull Lake Dr.

7 Hickory Corners, MI 49060

8 USA

9 and

10 Five Rivers Services at

11 US Geological Survey

12 National Wetland Research Center

13700 Cajundome Blvd.

14 Lafayette, LA 70506

15 USA 
The potential of spatial heterogeneity to promote plant species coexistence is well

19 documented theoretically (reviewed in Amarasekare 2003), but empirical support documenting

20 the power and scope of its ability to support diverse natural communities is mixed. For example,

21 Lundholm (2009) reviewed 41 observational studies and 11 experimental studies that quantified

22 the relationship between plant species diversity and spatial environmental heterogeneity and

23 found that, while many studies documented positive relationships between the two, the cross-

24 study effect size was not significantly different from zero.

One potential reason for the uncertainty observed in the relationship between plant

26 species diversity and spatial environmental heterogeneity is that the strength of the effect

27 depends on what aspect of the environment is varying; specifically whether it is resource or non-

28 resource factors that vary over space. In experiments and observational studies where a non-

29 resource environmental factor (e.g. soil type, $\mathrm{pH}$ ) varies, positive relationships between spatial

30 heterogeneity and species diversity are often observed (Reynolds et al. 1997, and many more,

31 reviewed in Lundholm 2009). However, there is surprisingly little empirical support for strong

32 positive relationships between the degree of spatial heterogeneity in a limiting resource and plant

33 species diversity at the local scale (Stevens and Carson 2002, Bakker et al. 2003, Reynolds et al.

34 2007, Lundholm 2009). For example, categorizing the factors in the studies reviewed by

35 Lundholm (2009) based upon whether they are resource or non-resources factors, reveals that a

36 significant relationship was found between species diversity and spatial heterogeneity for $71 \%$ 
37 (49 of 69) of non-resource factors, but only $28.5 \%$ (2 of 7 ) of resource factors ${ }^{1}$. These results

38 suggest an important question: should we expect that resource variation should have the same

39 effect on diversity as non-resource variation?

To understand why resource heterogeneity may less effectively facilitate species diversity

41 requires insight into how these factors affect variation dependent species coexistence

42 mechanisms (Chesson 2000a). If plant species are competing for a common resource, then in a

43 uniform environment, the species that can maintain a positive growth rate at the lowest resource

44 concentration (lowest $R^{*}$ Tilman 1982) is expected to drive all others to extinction. If spatial

45 environmental heterogeneity is to facilitate species coexistence, it must cause variation over

46 space in the identity of the species that has lowest $R^{*}$ (Amarasekare 2003). For the case of non-

47 resource spatial heterogeneity, Chesson (2000a) has identified the three variation dependent

48 coexistence mechanisms that cause variation in competitive ability over space and thus facilitate

49 coexistence: 1) spatial relative non-linearitiy, which can occur if species have different non-

50 linear responses to a common competitive environment. 2) growth-density covariance, which

51 measures a species ability to concentrate its population in the areas that best promote growth (in

52 the absence of competition), and 3) spatial storage effect, which occurs when different species

53 experience best growth in different areas of the environment. Of these three mechanisms, the

54 storage effect is potentially the most important in that it is evoked by many kinds of trait

55 differences among species (making it potentially common) and has been shown to allow the

56 coexistence of many species (Chesson 1994).

While others have shown that spatial variation is resource supply rates can facilitate

${ }^{1}$ Factors related to water (e.g. soil moisture) were left out of these tallies since they can often act as both resource and non-resource factors. 
58 coexistence, a similar partitioning of the mechanisms involved has not been reported. One

59 potential reason for the lack of attention to the difference in resource and non-resource spatial

60 variation is that prominent early theoretical papers made assumptions that minimized the

61 differences between them. For example, Tilman and Pacala (1993) and Abrams (1988) published

62 models that assumed the environment consists of discrete patches containing multiple limiting

63 resources, and that there is no, or only limited dispersal between patches, or that dispersal occurs

64 at a time-scale much slower than that of local competitive exclusion. These assumptions

65 approximate a situation where the spatial scale of heterogeneity is much larger than the

66 characteristic dispersal distances of the species in the community (i.e. most dispersal events

67 occur within patches, few between patches). Under these conditions, spatial heterogeneity creates

68 opportunities for species coexistence if each species is the best competitor at some ratio of

69 resource supply rates represented in a subset of patches (i.e., $R^{*}$ changes over space). This result

70 is similar to the general result obtained from models of non-resource, environmental

71 heterogeneity based coexistence (Chesson 2000a).

Recently however, researchers have begun studying models that assume that competitive

73 exclusion and dispersal occur over similar time-scales (Abrams and Wilson 2004, Golubski et al.

74 2008). This assumption approximates the case where the spatial scale of resource heterogeneity

75 is shorter than typical dispersal distances, and is probably more typical of the systems measured

76 in field studies. These models predict that a poor resource competitor may coexist with a better

77 resource competitor, if the better resource competitor experiences more inter-patch dispersal

78 (Abrams and Wilson 2004). Coexistence in this case is possible because dispersal results in a net

79 loss of individuals from the richest patches which in turn reduces the population's ability to

80 depress resource concentrations in those patches as low as it would in the absence of dispersal; 
81 allowing persistence of a competitor that experiences less inter-patch dispersal but has a higher

$82 \mathrm{R}^{*}$ (in a uniform environment). However, the identity and relative strength of the coexistence

83 mechanisms involved are yet to be quantified.

The assumption that competition and dispersal occur simultaneously increases the

85 complexity of the models, leading most researchers to model systems consisting of only a small

86 number of patches (Abrams and Wilson 2004, Golubski et al. 2008). As a result, these models

87 lack the generality that would allow them to be scaled up to quantify metacommunity-level

88 phenomena such as variation-dependent coexistence mechanisms (i.e. the storage-effect, relative

89 nonlinearity, and growth-density covariance, Chesson 2000a,b). In this article, I derive a simple

90 metacommunity model of plant competition for a single, spatially variable resource. I derive

91 approximate analytical relationships for regional species coexistence from which

92 metacommunity-scale population growth rates may be partitioned into the variation-dependent

93 and variation-independent coexistence mechanisms. These mechanisms are used to argue why

94 spatial variation for resources is less effective than non-resource spatial variation in facilitating

95 coexistence of many species.

The goal of this model is to answer the questions "how many species can coexist via

98 spatial heterogeneity in resource supply and by what means?” To answer these questions, I

99 define a simple model of plants growing in a spatially heterogeneous environment, and then use

100 the framework developed by Chesson (1994) to partition the regional growth rates implied by the

101 model into contributions from variation-independent and variation-dependent mechanisms. 
102

103

104

105

106

107

108

109

110

111

112

113

114

115

116

117

118

119

Finally, I use a sequential invasion approach with the mutual invasion criterion to determine how many species each mechanism allows to coexist at equilibrium.

In a set of discrete patches, $x=\{1,2,3, . ., N\}$, let $n_{j x}(t)$ be the density of species $j$ in patch $x$ at time $t$, and let $R_{x}(t)$ be the resource concentration in patch $x . n_{j x}$ decreases over time at the per capita mortality rate $m_{j}$, and increases by reproduction in the current patch, which occurs at the resource-dependent per-capita growth rate $b_{j} R_{x}$, plus the contributions of dispersal into patch $x$ from other patches. Let $p_{j}$ be the proportion of seeds produced by adults of species $j$ in any patch that remain in that patch and assume that the $1-p_{j}$ seeds that leave natal patches are evenly redistributed among all patches (including the natal patch). Resources are increased in patches at a constant rate $S_{x}$ and are reduced through the establishment of plants. The resource model is kept intentionally simple to allow analytical treatment. The inclusion of additional loss terms for resources, for example to adult plant maintenance or leaching do not affect the conclusions (Supplemental Appendix S2). The dynamics of this coupled system are described by,

$$
\begin{aligned}
& \frac{d n_{j x}}{d t}=-m_{j} n_{j x}+b_{j} R_{x}\left(p_{j} n_{j x}+\left(1-p_{j}\right)\left\langle n_{j}\right\rangle_{x}\right) \\
& \frac{d R_{x}}{d t}=S_{x}-\sum_{j} Q_{j} b_{j} R_{x}\left(p_{j} n_{j x}+\left(1-p_{j}\right)\left\langle n_{j}\right\rangle_{x}\right)
\end{aligned} .
$$

In eqn $1, Q_{j}$ is the amount of resource required for establishment; $S_{x}$ is the patch-specific resource supply rate and $\langle\cdot\rangle_{x}$ indicates a mean taken over patches. For a single species, this system has one stable equilibrium point per patch at, 
120 where the $\mathrm{m}$ in $R_{j x}^{* m}$ is used to differentiate this equilibrium concentration of resource from the

121 traditional $R^{*}$, that occurs in an uncoupled or homogenous system and is independent of

122 resource supply rate.

123 Eqn.2 shows that, in this model, dispersal has no effect on equilibrium density, $n_{j x} *$.

124 However, the equilibrium resource concentration, $R_{j x}^{* m}$ does depend on the amount of dispersal

125 between patches and the resource supply rate in patch $x$ relative to the mean supply rate in the

126 metacommunity. Specifically, if dispersal between patches is high, species $j$ leaves a higher

127 concentration of resources behind in patches with above average supply rates and a lower

128 concentration in patches with below average supply rates than it would in a homogenous

129 environment. This occurs because patches with high supply rates are net exporters of recruits and

130 patches with low supply rates are net importers of potential recruits. The increased concentration

131 of available resources in high supply rate patches allows the invasion and possible coexistence of

132 a species that has a higher $R^{*}$, but experiences less inter-patch dispersal (Abrams and Wilson

133 2004)(Fig. 1).

134 Derivation of variation dependent mechanisms.

135 One way to measure potential for coexistence is with the mutual invasion criterion. This

136 criterion states that a set of species can coexist with one another if each can invade the

137 equilibrium assemblage of the other species in the set. In practice, one calculates growth rate of

138 a species with inter-specific competition set at the value determined by the competitors at

139 equilibrium and intra-specific competition set to zero. This is called the low-density growth rate

140 of the species. In a spatial context, we are interested in coexistence at the larger scale of the set 
141 of all patches, so we calculate what is called the low-density metacommunity scale (or regional)

142 growth rate.

143 Chesson (2000a) has shown how a low density metacommunity-scale growth rate can be

144 calculated an written in terms of variation dependent coexistence mechanisms. First, the local

145 growth rate is decomposed into terms quantifying the direct effects of environmental variation

$146(E)$, variation in competition $(C)$ and their interaction

$$
r_{j x}=\mathscr{E}_{x}-\mathscr{C}_{j x}+\gamma_{j} \mathscr{E}_{x} \mathscr{C}_{j x}
$$

148 where $\mathscr{C}_{x}=G_{j}\left(E_{x}, C_{j}^{*}\right), \mathscr{C}_{j x}=-G\left(E^{*}, C\right), \gamma_{j}=\frac{\partial G_{j}}{\partial \mathscr{C} \partial \mathscr{C}_{j}}$.

149 The quantities $E$ and $C$ are the population parameters affected by environmental variation and the 150 effect of competition (which is also affected by environmental variation) respectively, $G$ is the 151 growth rate of species $j$ as a function of $E$ and $C$ and the $(*)$ indicates the equilibrium level of the 152 value.

153 The metacommunity-scale growth rate of species $j, \widetilde{r}_{j}$, in a spatially heterogeneous environment

154 is found by taking the mean of $r_{j x}$ over all individuals in the metapopulation, $\tilde{r}_{j}=\frac{1}{\sum_{x} n_{j x}} \sum_{x} r_{j x} n_{j x}$.

155 It can be written in terms of a spatial mean by defining relative local density as $v_{j x}=\frac{n_{j x}}{\left\langle n_{j}\right\rangle_{x}}$.

156 Substituting $v_{j x}$ into $\tilde{r}_{j}$ gives $\tilde{r}_{j}=\left\langle r_{j} v_{j}\right\rangle_{x}$, which can be rewritten as $\tilde{r}_{j}=\left\langle r_{j}\right\rangle_{x}+\operatorname{cov}\left(r_{j}, v_{j}\right)_{x}$,

157 where $\operatorname{cov}(.)_{\mathrm{x}}$ indicates a spatial covariance (Chesson 2000a). Plugging eqn 3 into this result 158 gives, 


$$
\widetilde{r_{j}}=\langle\mathscr{E}\rangle_{x}-\left\langle\mathscr{C}_{j}\right\rangle_{x}+\gamma_{j}\left\langle\mathscr{E} \mathscr{C}_{j}\right\rangle_{x}+\operatorname{cov}\left(r_{j}, v_{j}\right)_{x}
$$

160 To argue that any of the terms in eqn 4 contribute to coexistence, the difference between

161 invader's and residents’ values must be positive. Because, by definition the metacommunity

162 growth rate of the resident is zero, it can be subtracted from the right side of eqn 4 without

163 changing the left side. Thus, the metacommunity growth rate of the invader, denoted by subscript

$164 i$, can be rewritten in terms of contributions from multiple coexistence mechanisms by

165 subtracting the metacommunity growth rate of the residents, denoted by subscript $r$. Subtracting

166 the metacommunity growth rate of the residents gives,

$$
\tilde{r}_{i}=\Delta E-\Delta C+\Delta I+\Delta \kappa
$$

168 where

$$
\begin{aligned}
& \Delta E=\left\langle\mathscr{C}_{i}\right\rangle_{x}-q_{i r}\left\langle\mathscr{E}_{r}\right\rangle_{x} \\
& \Delta C=\left\langle\mathscr{C}_{i}\right\rangle_{x}-q_{i r}\left\langle\mathscr{C}_{r}\right\rangle_{x} \\
& \Delta I=\gamma_{i}\left\langle\mathscr{E}_{r} \mathscr{C}_{i}\right\rangle_{x}-q_{i r} \gamma_{r}\left\langle\mathscr{E}_{r} \mathscr{C}_{r}\right\rangle_{x} \\
& \Delta \kappa=\operatorname{cov}\left(r_{i}, v_{i}\right)_{x}-q_{i r} \operatorname{cov}\left(r_{r}, v_{r}\right)_{x}
\end{aligned}
$$

170 and the scaling factor is $q_{i r}=\frac{\partial \mathscr{C}_{i}}{\partial \mathscr{C}_{r}}$ is chosen to make the resulting expression more biologically

171 interpretable. For example, in this case, it allows $\Delta C$ to be expressed as a difference in the $R^{*}$ 172 values of the species.

The quantity $\Delta E$ measures differences in the average environment experienced by the

174 invader and resident. The quantity $\Delta C$ can contain both the fluctuation independent difference of

175 the average competition experienced by residents and invaders and a measure of the effect of

176 variation in competition. The combination $\Delta E-\Delta C$ is often rewritten to separate it into variation 
177 dependent $\Delta N$ and variation independent $\tilde{r}_{i}{ }^{\prime}$ parts, where $\tilde{r}_{i}{ }^{\prime}=\Delta E-\mathscr{C}_{i}{ }^{*}$ and $\Delta N=\Delta C-\mathscr{C}_{i}{ }^{*}$. The

178 quantity $\mathscr{C}_{i}^{*}$ is the value of competition the invader experiences as a consequence of the resource

179 equilibrium the residents create (Chesson 2000a). The quantity $\tilde{r}_{i}^{\prime}$ the growth rate the invader

180 would experience in the absence of variation. The quantity $\Delta N$ has been called relative-

181 nonlinearity and can facilitate coexistence if species exhibit different non-linear responses in

182 growth rate to variations in competition, specifically if the species with the larger non-linearity in

183 response experiences lower variance in competition. For most models that would describe the

184 growth of plants, this mechanism can only facilitate coexistence of two species (Chesson 1994).

185 The quantity $\Delta I$ is the storage effect (Chesson 1994) it measures the covariance between

186 the direct effect of environmental variation and the effect of competition on the growth rate of

187 the invader. This mechanism is potentially very powerful and can facilitate coexistence on many

188 species. An example of many species coexistence via spatial storage effect would where there

189 are many patches and each species is the best competitor in at least one patch (Sears and Chesson

190 2007).

The final mechanism, $\Delta \kappa$, is growth-density covariance. It measures the ability of the

192 invader to concentrate its population into patches that are best at supporting growth. The species

193 that is better able to do this will experience an overall boost to metacommunity scale growth rate.

194 This mechanism is most directly related to dispersal.

195 Derivation of variation dependent mechanisms for spatial resource heterogeneity

196 Following Chesson (2000), the model (eqns. 1) can be written in terms of variation in

197 environment, $E_{x}$ (the life history character that varies in space) and competition, $C_{x}$. In the case 
198 of the present model, where only the supply rate of resources varies over space, environmental

199 variation does not directly affect individuals, it only affects competition; thus $E_{x}=0$ and

$200 C_{x}=-R_{x}$. Making these substitutions, the model in eqns. 1can be rewritten as,

201

$$
\begin{aligned}
& \frac{d n_{j x}}{d t}=-m_{j} n_{j x}-b_{j} C_{x}\left(p_{j} n_{j x}+\left(1-p_{j}\right)\left\langle n_{j}\right\rangle_{x}\right) \\
& \frac{d C_{x}}{d t}=-S_{x}+\sum_{j} Q_{j} b_{j} C_{x}\left(p_{j} n_{j x}+\left(1-p_{j}\right)\left\langle n_{j}\right\rangle_{x}\right)
\end{aligned}
$$

203 The local growth rate is then, $r_{j x}=-m_{j}-b_{j} p_{j} C_{x}-\left(1-p_{j}\right) b_{j}\langle C\rangle_{x}$. Notice that in this case, due to

204 dispersal between patches $r_{j x} \neq \frac{1}{n_{j x}} \frac{d n_{j x}}{d t}$. Instead, $r_{j x}$ is derived by considering the fitness of an

205 individual in patch $x$ (Miller and Chesson 2009). Taking the mean over space, as described in the 206 previous section gives the metacommunity-scale growth rate, $\tilde{r}_{i}=-\left\langle\mathscr{C}_{i}\right\rangle_{x}+\operatorname{cov}\left(r_{i}, v_{i}\right)_{x}$, where $207\left\langle\mathscr{C}_{i}\right\rangle_{x}=m_{i}+b_{i}\langle C\rangle_{x}$. Assuming a single resident, denoted by subscript $r$, allows calculation of the 208 various coexistence mechanisms for the case of two species. This is helpful to determining the 209 presence or absence of the various mechanisms implied by this model. Plugging into eqn 6 and 210 scaling by $b_{i}$ to put the results in the natural time scale of generations instead of an absolute time 211 scale (e.g. months or years) (Chesson 2008) gives (for details see Supplemental Appendix S1), 


$$
\begin{aligned}
& \frac{\Delta E}{b_{i}}=0 \\
& \frac{\Delta C}{b_{i}}=-\frac{m_{i}}{b_{i}}+\frac{m_{r}}{b_{r}}
\end{aligned}
$$

$$
\frac{\Delta I}{b_{i}}=0
$$

$$
\frac{\Delta \kappa}{b_{i}} \approx \frac{m_{r}}{b_{r}}\left(1-p_{r}\right)^{2}\left\langle s^{2}\right\rangle_{x}\left(\frac{p_{i}}{1-p_{i}}-\frac{p_{r}}{1-p_{r}}\right) .
$$

213 Rewriting $\Delta E-\Delta C$ terms of $\tilde{r}_{i}^{\prime}$ and $\Delta N$ gives,

$$
\begin{aligned}
& \frac{\tilde{r}_{i}^{\prime}}{b_{i}}=-R_{i}^{*}+R_{r}^{*}, \\
& \frac{\Delta \kappa}{b_{i}} \approx \operatorname{var}(s)_{x}\left(1-p_{r}\right)^{2} R_{r}^{*}\left(\frac{p_{i}}{1-p_{i}}-\frac{p_{r}}{1-p_{r}}\right) .
\end{aligned}
$$

215 where $\frac{m_{j}}{b_{j}}=R_{j}^{*}$. Notice in this case $\Delta N=0$ since $\Delta C$ contains no variation dependent terms.

216 Biologically, $\tilde{r}_{i}{ }^{\prime} / b_{i}$ is a comparison of abilities to reduce resources in a homogenous

217 environment. Specifically, eqn 9 states that invaders are benefited by the ability to reduce

218 resources to a lower level than the resident in a homogenous environment (Tilman 1982). It also

219 suggests that in the absence of spatial heterogeneity (var $\left.(s)_{x}=0\right)$ coexistence is impossible

220 because only the species with the lowest $R^{*}$ would have a positive growth rate as an invader.

221 In addition, eqn. 9 shows that only differences in dispersal can allow coexistence in this

222 model. The storage effect $(\Delta I)$ and relative nonlinearity $(\Delta N)$ have no effect on invader’s

223 metacommunity-scale growth rate and thus do not contribute to coexistence. The effect of

224 growth-density covariance ( $\Delta \kappa)$ on the growth rate of the invader depends upon $R_{r}^{*}$ times the

225 odds of seeds staying in natal patches relative to the resident's. Invaders whose seeds are less 
226 widely dispersed than those of the resident species are benefited by growth-density covariance.

227 Thus, this model allows coexistence of species if there is a tradeoff competitive ability $\left(R^{*}\right)$ and

228 dispersal fraction (p). In addition, coexistence is facilitated by only one variation-dependent

229 mechanism, grow-density covariance.

230 How many species does growth-density covariance support?

231 I used a sequential invasion approach based on adaptive dynamics (Geritz et al. 1998) to

232 determine how many species can coexist based on this model. This approach attempts to find the

233 trait values for invaders that allow invasion in a given context. In this case, at each step, I find

234 the values for the trait $p_{i}$ that allows an invader to have a positive meta-community growth rate

235 (often written as $S\left(p_{i}\right)$ in the adaptive dynamics literature) in an assemblage of $n$ resident species,

236 i.e., $S_{\left\{p_{r m}\right\}}\left(p_{i}\right)>0$. If this species can 1) coexist with the current resident strategies and resist

237 exclusion by similar strategies (i.e. similar values $p$ ), then it is added the list of residents. Species

238 are added one at a time until there is no value of $p_{i}$ that leads to positive metacommunity-scale

239 growth rate. Because the approximated expression for growth-density covariance from eqn. 9

240 assumes small variation in supply rate and has singularities at $p_{i}=1$ and $p_{r}=1$, I use simulation of

241 eqn 1 to calculate invasion growth rate. Numerical simulations of the residents were run until

242 they reached a steady state. The resulting value of resource concentration was plugged into the

243 matrix that described each patches' contribution to an invader's low-density growth-rate in patch

$244 x$. The dominant eigenvalue of this matrix is an estimate of the meta-community scale low-

245 density growth rate of the invader. For the simulations, I assumed Log-normal distributed supply

246 rates and trade-offs between $R_{j}^{*}$ and $p_{j}$ of the form $R_{j}^{*}-p_{j}^{\tau}=Z$ where $Z$ is an arbitrary constant 
247 and $\tau$ affects the shape of the trade-off. The following example considers a linear tradeoff, 248 between competitive ability and dispersal i.e., $\tau=1$.

249 Figure 2 shows a contour plot for the invasibility of strategy $p_{i}$ in the presence of resident $p_{r}$. The 250 black region of Figure 2 represent negative invader growth rates (i.e. the invasion is 251 unsuccessful), the white regions shows areas of positive growth rates. It shows that any resident 252 strategy $p_{r}<1$ can be invaded by $p_{i}=1$. However, the strategy $p_{r}=1$ is also able to be invaded

253 by any $p_{i}<1$. Thus, I set $p_{r 1}=1$ and looked for values of $p_{r 2}$ that could coexist with $p_{r 1}$ and were 254 not excluded by other similar strategies. Figure 3 shows that given $p_{r 1}=1$, any resident strategy $255 p_{r 2}>0$ can be invaded by $p_{i}<p_{r 2}$. Thus, since $p_{r 2}=0$ can also coexist with $p_{r 1}=1$, it was added to the 256 resident list. Figure 3 also suggests that there is no third strategy $p_{i}$ that can coexist with $p_{r 1}=1$ 257 and $p_{r 2}=0$, since $S_{\left\{p_{r 11}, p_{r 2=0}\right\}}\left(p_{i}\right)<0$ for all possible values of $p_{i}$ (i.e. a vertical line drawn from $258 p_{r 2}=0$ passes only through the black region of the graph). This suggests that in this model, a 259 tradeoff between competitive ability $R_{j}^{*}$ and dispersal $p_{j}$, allows coexistence of only two species.

260 To assure these results were robust, I simulated systems of simultaneous competition among 261 many species from along the trade-off manifold (Supplemental Appendix S2). Those

262 simulations also show an observed maximum of two coexisting species for nonlinear tradeoffs 263 with $\tau<1$; additional loss terms in the resource equation, and saturating growth responses. For 264 non-linear tradeoffs with $\tau>1$ no coexistence was possible.

\section{Discussion}

267 mechanisms and lower potential species diversity compared to spatial variation in non-resource 268 factors. Although spatial resource variation promotes coexistence if there is a tradeoff between 
269

270

271

272

273

274

275

276

277

278

279

280

281

282

283

284

285

286

287

288

289

290

competitive ability and the ability to retain offspring in good patches, this tradeoff allows the coexistence of only a 2 species.

Coexistence under spatial resource variation lacks contributions from the spatial storage effect, a powerful mechanism that allows the coexistence of many species. The storage effect is absent because all species grow best in the same patches (high resource patches). As a result, environmental responses and competition are perfectly and equally correlated for all species, allowing no advantages in good patches when a species is rare. In other words, the feedback that organisms have on the resource, make it impossible for any to specialize on a particular supply rate along a gradient.

Although the mechanism of resource competition analyzed in this model is simplified, the qualitative results are quite general. For example, the relationship of $R^{*}$ to the metacommunity-scale coexistence mechanisms described by scale transition theory does not depend on the simplified model of local resource competition presented in eqns 1 . If the establishment rate of seeds is a saturating function (e.g. Monod functions with equal half saturation constants) of the amount of resources present, then $\widetilde{r}$ 'is still a comparison of the (more complicated) $R^{*}$ s of the invader and resident, and the relative importance of the variation dependent mechanism is the same. The situation is more complex if species growth rates are different non-linear functions of resource concentration, but, even in this case, spatial variation in one only facilitate 2 species coexistence (Supplemental Appendix S2).

In this model, competition was for a single resource. Increasing the number of limiting resources can increase the number of species that can coexist at equilibrium. Golubski et al. (2008) found that a maximum of four species could coexist in a system with two resources, 
291 heterogeneous supply rates and species that were capable of integrating growth across patches.

292 However, this was only possible with precise arrangement of species trait parameters, and if 293 resource supply rates of the resources were strongly negatively correlated across patches. I

294 presume that, in this case, adding resource factors does not increase the number of mechanisms;

295 the existing mechanism, growth-density covariance, simple works independently for each

296 resource; although this claim should be explored with further analysis.

This analysis suggests that spatial resource heterogeneity is not capable of supporting the 298 robust stable coexistence of many plants species that is observed in many natural systems. The

299 reason for this is that the feedback species have on resources concentrations prevents

300 specialization of different species at different supply rates. It is this kind of specialization of

301 different species along different points of a "niche" axis, which is measured by the spatial

302 storage effect, that allows the robust coexistence of many species. The results of this analysis are

303 consistent with the patterns found in the empirical literature, which finds much more support for

304 the relationship between species diversity and nonresource spatial heterogeneity than resource

305 heterogeneity. Taken together, this work suggests that the consideration whether a factor is a

306 resource or not is crucial for those attempting to understand real patterns in species diversity or

307 those interested in managing a habitat for increased species diversity.

308

Acknowledgements

309 I would like to thank Peter Chesson, Kay Gross, and Gary Mittelbach for helping improve this

310 manuscript. This is contribution No. TBD from the W.K. Kellogg Biological Station, Michigan

311 State University.

312

References

16 
314 Abrams, P.A. 1988. Resource productivity - consumer species diversity: simple models of 315 competition in spatially heterogeneous environments. Ecology 69: 1418-1433.

316 Abrams, P.A., W.G. Wilson. 2004. Coexistence in metacommunities due to spatial variation in 317 resource growth rates; does $\mathrm{R}^{*}$ predict the outcome of competition? Ecology Letters 7:929-940.

318 Amarasekare, P. 2003. Competitive coexistence in spatially structured environments: a synthesis. 319 Ecology Letters 6: 1109-1122.

320 Bakker, C., Blair, J.M., Knapp, A.M. 2003. Does resource availability, resource heterogeneity or 321 species turnover mediate changes in plant species richness in grazed grasslands? Oecologia 137: 322 385-391.

Chesson, P. 1994. Multispecies competition in variable environments. Theoretical Population Biology 45: 227-276.

Chesson, P. 2000a. General theory of competitive coexistence in spatially-varying environments.

326 Theoretical Population Biology 58: 211-237.

Chesson, P. 2000b. Mechanisms of maintenance of species diversity. Annual Review of Ecology, Evolution \& Systematics 31: 343-66.

Chesson, P. 2008. Quantifying and testing species coexistence mechanisms.-- In: F. Valladares, A. Camacho, A. Elosegui, C. Gracia, M. Estrada, J. C. Senar, and J. M. Gili, eds. Unity in Diversity: Reflections on Ecology after the Legacy of Ramon Margalef. Fundacion BBVA, Bilbao. pp. 119-164

S. A. H. Geritz, E. Kisdi, G. Meszena, J. A. J. Metz. 1998., Evolutionarily singular strategies and the adaptive growth and branching of the evolutionary tree, Evolutionary Ecology, 12:, 35-57.

Golubski, A.J., Gross, K.L., Mittelbach, G.G. 2008. Competition among plant species that interact with their environment at different spatial scales. Proceeding of Royal Society, $B$ 275:1897-906.

339 Lundholm, J.T. 2009. Plant Species diversity and environmental heterogeneity: spatial scale and competing hypothesis. Journal of Vegetation Science 20: 377-391.

341 Miller, A. D., Chesson, P. 2009. Coexistence in disturbance prone communities: how a 342 resistance-resilience trade off generates coexistence via the storage effect. The American 343 Naturalist 173, E30-E43.

344 Reynolds, H.R., Hungate, B.A., Chapin III, F.S., D’Antonio, C. 1997. Soil heterogeneity and 345 plant competition in an annual grassland. Ecology 78: 2076-2090.

346 Reynolds, H.R., Mittelbach, G.G., Darcy-Hall, T., Houseman, G.R., Gross, K.L. 2007. No effect 
347 of varying soil resource heterogeneity on plant species richness in a low fertility grassland.

348 Journal of Ecology, 95: 723-733.

349 Sears, A.L.W, Chesson, P. 2007. New methods for quantifying the spatial storage effect: an 350 illustration with desert annuals. Ecology 88: 2240-2247.

351 Stevens, M.H.H., Carson, W. 2002. Resource quantity, not resource heterogeneity maintains 352 plant diversity. Ecology Letters: 5: 420-426.

353 Tilman, D. 1982. Resource Competition and Community Structure. Princeton University Press.

354 Tilman, D., Pacala,S. 1993. The maintenance of species richness in plant communities. - In: 355 Ricklefs, R. and Schluter, D., eds. Species Diversity in Ecological Communities, University of 356 Chicago Press, Chicago. pp. 13-25. 


\section{Figure 1}

$\mathrm{R}^{*}$ as a function of dispersal

Concentration of resources left behind at equilibrium $\left(\$ \mathrm{R}^{\wedge}\left\{{ }^{*} \mathrm{~m}\right\} \_\{\mathrm{jx}\}\right)$ along a spatial gradient of resource supply rates. The $\$ R^{\wedge}\left\{{ }^{*} m\right\} \_\{j x\} \$$ of a species depends on the supply rate of the patch and the amount of interpatch dispersal (Black lines: $R^{*}=0.4, p=1$, solid; $p=0.66$, dashed; $p=0.33$, dash-dot; $p=0$, dotted). As a result, a species with a higher $R^{*}$ may invade the metacommunity if it experiences less interpatch dispersal (Grey line: $R^{*}=0.5 ; \mathrm{p}=0.9$ ) because it can have a $\$ \mathrm{R}^{\wedge}\{* \mathrm{~m}\}_{-}\{\mathrm{jx}\} \$$ lower [i] in patches with the highest supply rates.

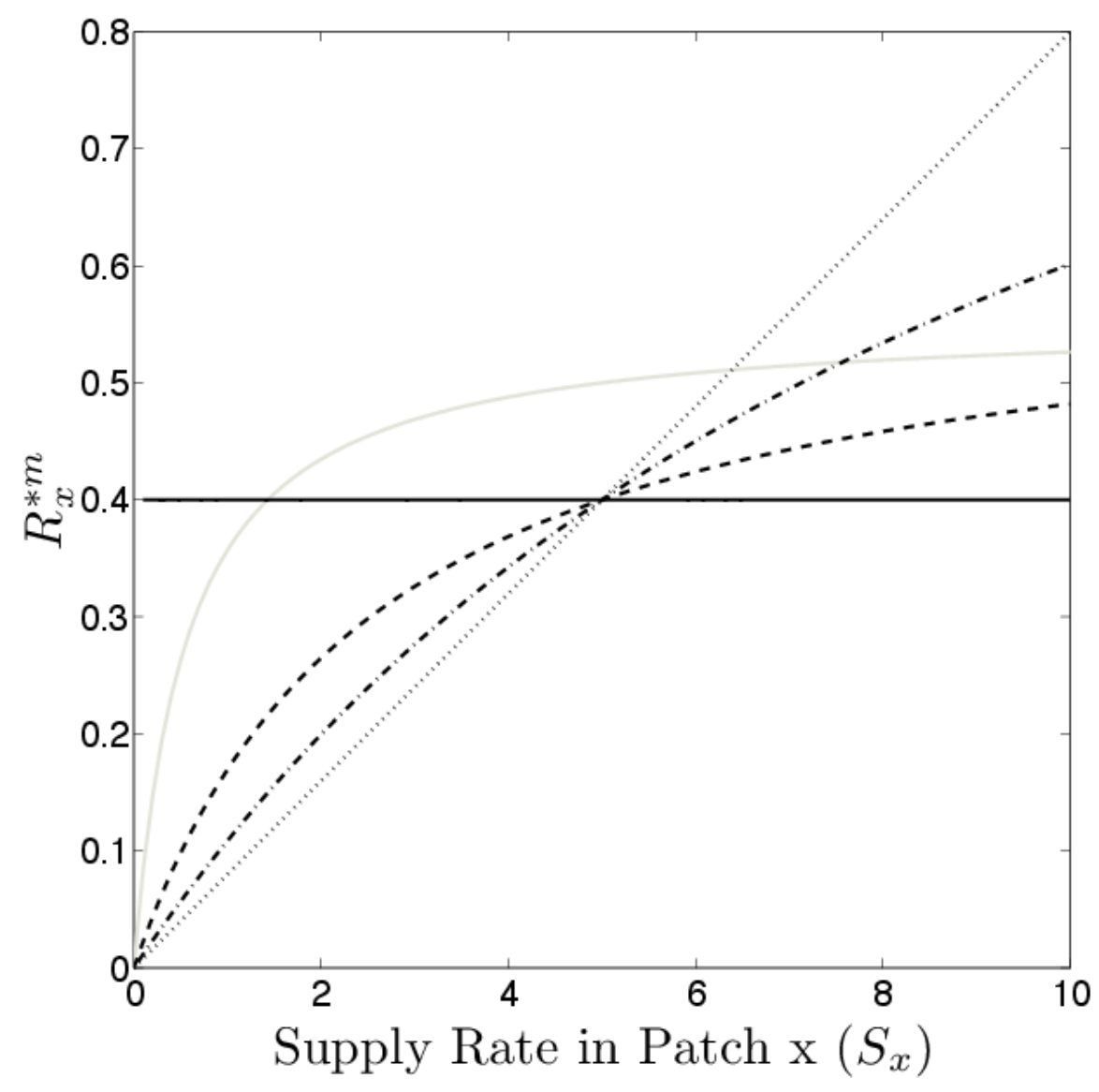




\section{Figure 2}

Invasion analysis

Contour plot of the growth rate of an invader with dispersal $p_{i}$ as a function of resident dispersal $p_{r}$, assuming a tradeoff between competitive ability $\left(R^{*}\right)$ and dispersal $(p)$. Black regions show areas of negative invader growth rate; white regions, positive. The graph shows that $p_{i}=1$ can invade a meta-community with a resident with $p_{r}<1$. It also shows that $p_{r}=1$ can be invaded by any $p_{i}<1$, suggesting coexistence with this strategy is possible. Parameter values for this graph are $R_{j}^{*}=1-p_{j}$ in a system of 20 patches and $\mathrm{S}_{\mathrm{x}} \sim \operatorname{LogNormal}(1,1.5)$.

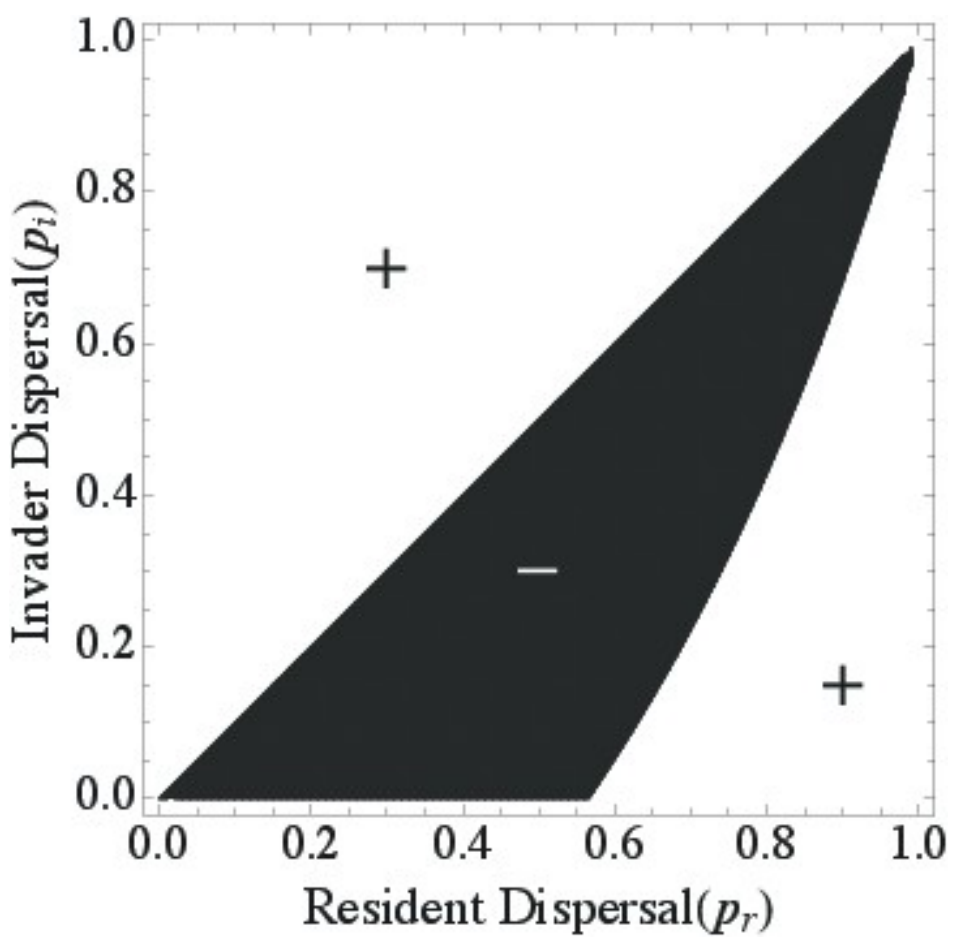




\section{Figure 3}

2 Species Invasion Analysis

Contour plot of the growth rate of an invader with dispersal $p_{i}$ as a function of resident dispersal $p_{r 1}=1, p_{r 2}$, assuming a tradeoff between competitive ability $\left(R^{*}\right)$ and dispersal $(p)$. Black regions show areas of negative invader growth rate; white regions, positive. The graph shows that given $p_{r 1}=1$, $p_{r 2}>0$ can be invaded by and excluded by $p_{i} r$. Thus, it shows that given $p_{r 1}=1$ and $p_{r 2}=0$, no third strategy $p_{i}$ has a positive growth rate; confirming that stable coexistence of only two species is possible. Parameter values for this graph are $R_{j}^{*}=1-p_{j}$ in a system of 20 patches and $\mathrm{S}_{\mathrm{X}} \sim \operatorname{LogNormal}(1,1.5)$.

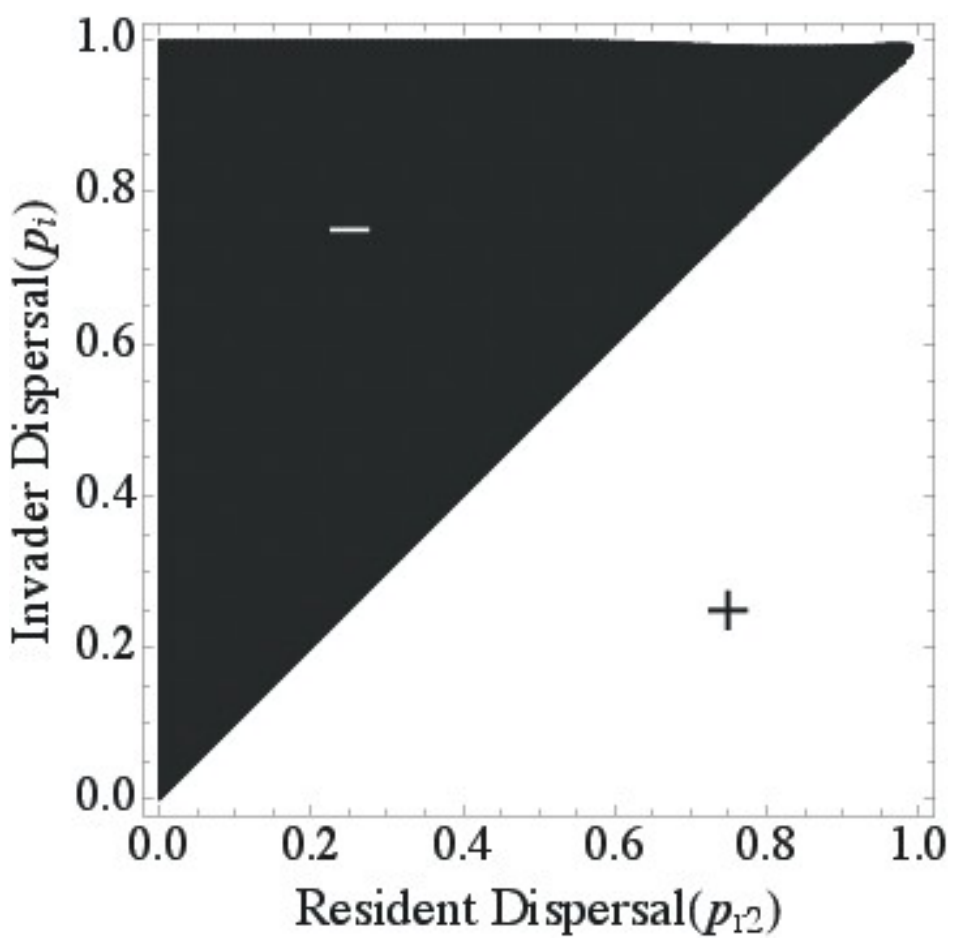


2 Donald R. Schoolmaster Jr.

3 schoolm4@msu.edu

4 W.K. Kellogg Biological Station

5 Michigan State University

63700 E. Gull Lake Dr.

7 Hickory Corners, MI 49060

8 USA

9 and

10 Five Rivers Services at

11 US Geological Survey

12 National Wetland Research Center

13700 Cajundome Blvd.

14 Lafayette, LA 70506

15 USA 
The potential of spatial heterogeneity to promote plant species coexistence is well documented theoretically (reviewed in Amarasekare 2003), but empirical support documenting the power and scope of its ability to support diverse natural communities is mixed. For example, Lundholm (2009) reviewed 41 observational studies and 11 experimental studies that quantified the relationship between plant species diversity and spatial environmental heterogeneity and found that, while many studies documented positive relationships between the two, the cross-study effect size was not significantly different from zero.

One potential reason for the uncertainty observed in the relationship between plant species diversity and spatial environmental heterogeneity is that the strength of the effect depends on what aspect of the environment is varying; specifically whether it is resource or non-resource factors that vary over space. In experiments and observational studies where a non-resource environmental factor (e.g. soil type, $\mathrm{pH}$ ) varies, positive relationships between spatial heterogeneity and species diversity are often observed (Reynolds et al. 1997, and many more, reviewed in Lundholm 2009). However, there is surprisingly little empirical support for strong positive relationships between the degree of spatial heterogeneity in a limiting resource and plant species diversity at the local scale (Stevens and Carson 2002, Bakker et al. 2003,

34 Reynolds et al. 2007, Lundholm 2009). For example, categorizing the factors in the studies reviewed by Lundholm (2009) based upon whether they are resource or non-resources factors, reveals that a significant relationship was found between species diversity and spatial

37 heterogeneity for $71 \%$ (49 of 69) of non-resource factors, but only $28.5 \%$ (2 of 7 ) of resource 
38 factors ${ }^{1}$. These results suggest an important question: should we expect that resource variation

39 should have the same effect on diversity as non-resource variation?

40 To understand why resource heterogeneity may less effectively facilitate species diversity

41 requires insight into how these factors affect variation dependent species coexistence

42 mechanisms (Chesson 2000a). If plant species are competing for a common resource, then in a

43 uniform environment, the species that can maintain a positive growth rate at the lowest resource

44 concentration (lowest $R^{*}$ Tilman 1982) is expected to drive all others to extinction. If spatial

45 environmental heterogeneity is to facilitate species coexistence, it must cause variation over

46 space in the identity of the species that has lowest $R^{*}$ (Amarasekare 2003). For the case of

47 non-resource spatial heterogeneity, Chesson (2000a) has identified the three variation dependent

48 coexistence mechanisms that cause variation in competitive ability over space and thus facilitate

49 coexistence: 1) spatial relative non-linearitiy, which can occur if species have different non-linear

50 responses to a common competitive environment. 2) growth-density covariance, which measures

51 a species ability to concentrate its population in the areas that best promote growth (in the

52 absence of competition), and 3) spatial storage effect, which occurs when different species

53 experience best growth in different areas of the environment. Of these three mechanisms, the

54 storage effect is potentially the most important in that it is evoked by many kinds of trait

55 differences among species (making it potentially common) and has been shown to allow the

56 coexistence of many species (Chesson 1994).

57 While others have shown that spatial variation is resource supply rates can facilitate

58 coexistence, a similar partitioning of the mechanisms involved has not been reported. One

11 Factors related to water (e.g. soil moisture) were left out of these tallies since they can often act

2 as both resource and non-resource factors. 
59 potential reason for the lack of attention to the difference in resource and non-resource spatial

60 variation is that prominent early theoretical papers made assumptions that minimized the

61 differences between them. For example, Tilman and Pacala (1993) and Abrams (1988) published

62 models that assumed the environment consists of discrete patches containing multiple limiting

63 resources, and that there is no, or only limited dispersal between patches, or that dispersal occurs

64 at a time-scale much slower than that of local competitive exclusion. These assumptions

65 approximate a situation where the spatial scale of heterogeneity is much larger than the

66 characteristic dispersal distances of the species in the community (i.e. most dispersal events

67 occur within patches, few between patches). Under these conditions, spatial heterogeneity creates

68 opportunities for species coexistence if each species is the best competitor at some ratio of

69 resource supply rates represented in a subset of patches (i.e., $R^{*}$ changes over space). This result

70 is similar to the general result obtained from models of non-resource, environmental

71 heterogeneity based coexistence (Chesson 2000a).

Recently however, researchers have begun studying models that assume that competitive

73 exclusion and dispersal occur over similar time-scales (Abrams and Wilson 2004, Golubski et al.

74 2008). This assumption approximates the case where the spatial scale of resource heterogeneity

75 is shorter than typical dispersal distances, and is probably more typical of the systems measured

76 in field studies. These models predict that a poor resource competitor may coexist with a better

77 resource competitor, if the better resource competitor experiences more inter-patch dispersal

78 (Abrams and Wilson 2004). Coexistence in this case is possible because dispersal results in a net

79 loss of individuals from the richest patches which in turn reduces the population's ability to

80 depress resource concentrations in those patches as low as it would in the absence of dispersal;

81 allowing persistence of a competitor that experiences less inter-patch dispersal but has a higher 
$82 \mathrm{R}^{*}$ (in a uniform environment). However, the identity and relative strength of the coexistence

83 mechanisms involved are yet to be quantified.

84 The assumption that competition and dispersal occur simultaneously increases the

85 complexity of the models, leading most researchers to model systems consisting of only a small 86 number of patches (Abrams and Wilson 2004, Golubski et al. 2008). As a result, these models

87 lack the generality that would allow them to be scaled up to quantify metacommunity-level 88 phenomena such as variation-dependent coexistence mechanisms (i.e. the storage-effect, relative 89 nonlinearity, and growth-density covariance, Chesson 2000a,b). In this article, I derive a simple 90 metacommunity model of plant competition for a single, spatially variable resource. I derive 91 approximate analytical relationships for regional species coexistence from which 92 metacommunity-scale population growth rates may be partitioned into the variation-dependent 93 and variation-independent coexistence mechanisms. These mechanisms are used to argue why 94 spatial variation for resources is less effective than non-resource spatial variation in facilitating 95 coexistence of many species. Model The goal of this model is to answer the questions "how many species can coexist via 98 spatial heterogeneity in resource supply and by what means?” To answer these questions, I define 99 a simple model of plants growing in a spatially heterogeneous environment, and then use the 100 framework developed by Chesson (1994) to partition the regional growth rates implied by the 101 model into contributions from variation-independent and variation-dependent mechanisms.

102 Finally, I use a sequential invasion approach with the mutual invasion criterion to determine how 103 many species each mechanism allows to coexist at equilibrium. 
105 from other patches. Let $p_{j}$ be the proportion of seeds produced by adults of species $j$ in any patch

$$
1-p_{j}
$$

109 that remain in that patch and assume that the seeds that leave natal patches are evenly

110 redistributed among all patches (including the natal patch). Resources are increased in patches at 111 a constant rate $S_{x}$ and are reduced through the establishment of plants. The resource model is kept

112 intentionally simple to allow analytical treatment. The inclusion of additional loss terms for

113 resources, for example to adult plant maintenance or leaching do not affect the conclusions

114 (Supplemental Appendix S2). The dynamics of this coupled system are described by,

$$
\begin{aligned}
& \frac{d n_{j x}}{d t}=-m_{j} n_{j x}+b_{j} R_{x}\left(p_{j} n_{j x}+\left(1-p_{j}\right)\left\langle n_{j}\right\rangle_{x}\right) \\
& \frac{d R_{x}}{d t}=S_{x}-\sum_{j} Q_{j} b_{j} R_{x}\left(p_{j} n_{j x}+\left(1-p_{j}\right)\left\langle n_{j}\right\rangle_{x}\right)
\end{aligned}
$$

\langle\rangle$_{x}$

117 resource supply rate and indicates a mean taken over patches. For a single species, this

118 system has one stable equilibrium point per patch at,

$$
n_{j x}^{*}=\frac{S_{x}}{Q_{j} m_{j}}, R_{j x}^{* m}=\frac{m_{j} S_{x}}{b_{j}\left(p_{j} S_{x}+\left(1-p_{j}\right)\langle S\rangle_{x}\right)}
$$


120 where the $\mathrm{m}$ in ${ }_{j x}^{* m}$ is used to differentiate this equilibrium concentration of resource from the

121 traditional $R^{*}$, that occurs in an uncoupled or homogenous system and is independent of

122 resource supply rate.

123 Eqn.2 shows that, in this model, dispersal has no effect on equilibrium density, $n_{j x} *$.

124 However, the equilibrium resource concentration, ${ }^{R_{j x}^{* m}}$ does depend on the amount of dispersal

125 between patches and the resource supply rate in patch $x$ relative to the mean supply rate in the

126 metacommunity. Specifically, if dispersal between patches is high, species $j$ leaves a higher

127 concentration of resources behind in patches with above average supply rates and a lower

128 concentration in patches with below average supply rates than it would in a homogenous

129 environment. This occurs because patches with high supply rates are net exporters of recruits and

130 patches with low supply rates are net importers of potential recruits. The increased concentration

131 of available resources in high supply rate patches allows the invasion and possible coexistence of

132 a species that has a higher $R^{*}$, but experiences less inter-patch dispersal (Abrams and Wilson

133 2004)(Fig. 1).

134 Derivation of variation dependent mechanisms.

135 One way to measure potential for coexistence is with the mutual invasion criterion. This

136 criterion states that a set of species can coexist with one another if each can invade the

137 equilibrium assemblage of the other species in the set. In practice, one calculates growth rate of

138 a species with inter-specific competition set at the value determined by the competitors at

139 equilibrium and intra-specific competition set to zero. This is called the low-density growth rate 
140 of the species. In a spatial context, we are interested in coexistence at the larger scale of the set

141 of all patches, so we calculate what is called the low-density metacommunity scale (or regional)

142 growth rate.

143 Chesson (2000a) has shown how a low density metacommunity-scale growth rate can be

144 calculated an written in terms of variation dependent coexistence mechanisms. First, the local

145 growth rate is decomposed into terms quantifying the direct effects of environmental variation

$146(E)$, variation in competition $(C)$ and their interaction

147

$$
r_{j x}=\underset{\bar{X}}{\mathcal{E}}-\mathcal{C}_{j x}+\gamma_{j} \underset{\overline{\mathcal{E}}}{\mathcal{E}} C_{j x}
$$

$$
\mathcal{E}_{x}=G_{j}\left(E_{x}, C_{j}^{*}\right) \quad C_{j x}=-G\left(E^{*}, C\right) \quad \gamma_{j}=\frac{\partial G_{j}}{\partial \mathcal{E} \partial C_{j}}
$$

148 where$$
\downarrow * \text { MERGEFORMAT () }
$$

149 The quantities $E$ and $C$ are the population parameters affected by environmental variation and the

150 effect of competition (which is also affected by environmental variation) respectively, $G$ is the

151 growth rate of species $j$ as a function of $E$ and $C$ and the $(*)$ indicates the equilibrium level of the

152 value.

153 The metacommunity-scale growth rate of species $j, \quad$, in a spatially heterogeneous environment

$r_{j x}$

$$
\hat{r}_{j}=\frac{1}{\sum_{x} n_{j x}} \sum_{x} r_{j x} n_{j x}
$$

154 is found by taking the mean of over all individuals in the metapopulation, 
155 It can be written in terms of a spatial mean by defining relative local density as

$$
v_{j x}=\frac{n_{j x}}{\left\langle n_{j}\right\rangle_{x}}
$$

\section{Substituting into gives}

$$
v_{j x} \quad r_{j} \quad \dot{r}_{j}=\left\langle r_{j} v_{j}\right\rangle_{x} \quad \hat{r}_{j}=\left\langle r_{j}\right\rangle_{x}+\operatorname{cov}\left(r_{j}, v_{j}\right)_{x}
$$

157 where $\operatorname{cov}(.)_{\mathrm{x}}$ indicates a spatial covariance (Chesson 2000a). Plugging eqn 3 into this result

158 gives,

$$
\dot{r}_{j}=\langle\mathcal{E}\rangle_{x}-\left\langle\mathcal{C}_{j}\right\rangle_{x}+\gamma_{j}\left\langle\mathcal{E} C_{j}\right\rangle_{x}+\operatorname{cov}\left(r_{j}, v_{j}\right)_{x}
$$

.${ }^{*}$ MERGEFORMAT ()

160 To argue that any of the terms in eqn 4 contribute to coexistence, the difference between

161 invader's and residents’ values must be positive. Because, by definition the metacommunity

162 growth rate of the resident is zero, it can be subtracted from the right side of eqn 4 without

163 changing the left side. Thus, the metacommunity growth rate of the invader, denoted by subscript

$164 i$, can be rewritten in terms of contributions from multiple coexistence mechanisms by

165 subtracting the metacommunity growth rate of the residents, denoted by subscript $r$. Subtracting

166 the metacommunity growth rate of the residents gives,

$$
\tilde{r}_{i}=\Delta E-\Delta C+\Delta I+\Delta \kappa
$$

168 where

$$
\begin{aligned}
& \Delta E=\langle\mathcal{F}\rangle_{x}-q_{i r}\left\langle\mathcal{F}_{T}\right\rangle_{x} \\
& \Delta C=\left\langle\mathcal{C}_{i}\right\rangle_{x}-q_{i r}\left\langle\mathcal{C}_{r}\right\rangle_{x} \\
& \Delta I=\gamma_{i}\left\langle\mathcal{E} C_{i}\right\rangle_{x}-q_{i r} \gamma_{r}\left\langle\mathcal{E} C_{r}\right\rangle_{x} \\
& \Delta \kappa=\operatorname{cov}\left(r_{i}, v_{i}\right)_{x}-q_{i r} \operatorname{cov}\left(r_{r}, v_{r}\right)_{x}
\end{aligned}
$$

।* MERGEFORMAT () 


$$
q_{i r}=\frac{\partial C_{i}}{\partial C_{r}}
$$

170 and the scaling factor is

is chosen to make the resulting expression more biologically

$\Delta C$

171 interpretable. For example, in this case, it allows to be expressed as a difference in the

172 values of the species.

173

$\Delta E$

The quantity measures differences in the average environment experienced by the

$\Delta C$

174 invader and resident. The quantity can contain both the fluctuation independent difference of

175 the average competition experienced by residents and invaders and a measure of the effect of

176 variation in competition. The combination $\Delta E-\Delta C$ is often rewritten to separate it into variation

177 dependent $\Delta N$ and variation independent $\tilde{r}_{i}^{\prime}$ parts, where $\tilde{r}_{i}^{\prime}=\Delta E-\mathcal{C}_{i}^{*} \Delta N=\Delta C-\mathcal{C}_{i}^{*}$ and . The

178 quantity $\mathcal{C}^{*}$ is the value of competition the invader experiences as a consequence of the resource

$\tilde{r}^{\prime}$

179 equilibrium the residents create (Chesson 2000a). The quantity the growth rate the invader

180 would experience in the absence of variation. The quantity $\Delta N$ has been called

181 relative-nonlinearity and can facilitate coexistence if species exhibit different non-linear

182 responses in growth rate to variations in competition, specifically if the species with the larger

183 non-linearity in response experiences lower variance in competition. For most models that 
184 would describe the growth of plants, this mechanism can only facilitate coexistence of two 185 species (Chesson 1994).

$\Delta I$ the direct effect of environmental variation and the effect of competition on the growth rate of 188 the invader. This mechanism is potentially very powerful and can facilitate coexistence on many 189 species. An example of many species coexistence via spatial storage effect would where there 190 are many patches and each species is the best competitor in at least one patch (Sears and Chesson 191 2007).

$\Delta \kappa$ The final mechanism, , is growth-density covariance. It measures the ability of the 193 invader to concentrate its population into patches that are best at supporting growth. The species 194 that is better able to do this will experience an overall boost to metacommunity scale growth rate. 195 This mechanism is most directly related to dispersal.

$$
E_{x} \quad C_{x}
$$

198 environment, (the life history character that varies in space) and competition, . In the case

199 of the present model, where only the supply rate of resources varies over space, environmental

200 variation does not directly affect individuals, it only affects competition; thus $E_{x}=0$

$$
C_{x}=-R_{x}
$$

201 . Making these substitutions, the model in eqns. 1can be rewritten as, 


$$
\begin{aligned}
& \frac{d n_{j x}}{d t}=-m_{j} n_{j x}-b_{j} C_{x}\left(p_{j} n_{j x}+\left(1-p_{j}\right)\left\langle n_{j}\right\rangle_{x}\right) \\
& \frac{d C_{x}}{d t}=-S_{x}+\sum_{j} Q_{j} b_{j} C_{x}\left(p_{j} n_{j x}+\left(1-p_{j}\right)\left\langle n_{j}\right\rangle_{x}\right)
\end{aligned}
$$

202

203

204 The local growth rate is then,

$$
r_{j x}=-m_{j}-b_{j} p_{j} C_{x}-\left(1-p_{j}\right) b_{j}\langle C\rangle_{x}
$$

. Notice that in this case, due to

205 dispersal between patches

$$
r_{j x} \neq \frac{1}{n_{j x}} \frac{d n_{j x}}{d t}
$$

206 individual in patch $x$ (Miller and Chesson 2009). Taking the mean over space, as described in the

$$
\tilde{r}_{i}=-\left\langle C_{i}\right\rangle_{x}+\operatorname{cov}\left(r_{i}, v_{i}\right)_{x}
$$

207 previous section gives the metacommunity-scale growth rate,

$$
\text { , where }
$$

$$
\left\langle C_{i}\right\rangle_{x}=m_{i}+b_{i}\langle C\rangle_{x}
$$

. Assuming a single resident, denoted by subscript $r$, allows calculation of the 209 various coexistence mechanisms for the case of two species. This is helpful to determining the 210 presence or absence of the various mechanisms implied by this model. Plugging into eqn 6 and 211 scaling by $b_{i}$ to put the results in the natural time scale of generations instead of an absolute time 212 scale (e.g. months or years) (Chesson 2008) gives (for details see Supplemental Appendix S1), 


$$
\begin{aligned}
& \frac{\Delta E}{b_{i}}=0 \\
& \frac{\Delta C}{b_{i}}=-\frac{m_{i}}{b_{i}}+\frac{m_{r}}{b_{r}} \\
& \frac{\Delta I}{b_{i}}=0 \\
& \frac{\Delta \kappa}{b_{i}} \approx \frac{m_{r}}{b_{r}}\left(1-p_{r}\right)^{2}\left\langle s^{2}\right\rangle_{x}\left(\frac{p_{i}}{1-p_{i}}-\frac{p_{r}}{1-p_{r}} \dot{\dot{j}}\right) \\
& 213 \\
& \text { I* MERGEFORMAT () } \\
& 214 \text { Rewriting } \Delta E-\Delta C \text { terms of } \tilde{r}_{i}^{\prime} \text { and } \Delta N \text { gives, } \\
& \begin{array}{l}
\frac{\tilde{r}_{i}^{\prime}}{b_{i}}=-R_{i}^{*}+R_{r}^{*}, \\
\frac{\Delta \kappa}{b_{i}} \approx \operatorname{var}(s)_{x}\left(1-p_{r}\right)^{2} R_{r}^{*}\left(\frac{p_{i}}{1-p_{i}}-\frac{p_{r}}{1-p_{r}}\right)
\end{array} \\
& 215 \\
& \text { I* MERGEFORMAT () } \\
& \frac{m_{j}}{b_{j}}=R_{j}^{*} \quad \text {. Notice in this case } \Delta N=0 \text { since } \Delta C \text { contains no variation dependent terms. } \\
& \tilde{r}_{i}{ }^{\prime} / b_{i}
\end{aligned}
$$

217 Biologically, is a comparison of abilities to reduce resources in a homogenous

218 environment. Specifically, eqn 9 states that invaders are benefited by the ability to reduce

219 resources to a lower level than the resident in a homogenous environment (Tilman 1982). It also

220 suggests that in the absence of spatial heterogeneity $\left(\operatorname{var}(s)_{x}=0\right)$ coexistence is impossible

221 because only the species with the lowest $R^{*}$ would have a positive growth rate as an invader.

223 model. The storage effect $(\stackrel{\Delta I}{ })$ and relative nonlinearity $(\stackrel{\Delta N}{\text { }})$ have no effect on invader's 
224 metacommunity-scale growth rate and thus do not contribute to coexistence. The effect of

225 growth-density covariance $\left({ }^{\Delta \kappa}\right.$ ) on the growth rate of the invader depends upon ${ }^{*}$ times the 226 odds of seeds staying in natal patches relative to the resident's. Invaders whose seeds are less 227 widely dispersed than those of the resident species are benefited by growth-density covariance. 228 Thus, this model allows coexistence of species if there is a tradeoff competitive ability $\left(R^{*}\right)$ and 229 dispersal fraction ( $p$ ). In addition, coexistence is facilitated by only one variation-dependent 230 mechanism, grow-density covariance.

231 How many species does growth-density covariance support?

232 I used a sequential invasion approach based on adaptive dynamics (Geritz et al. 1998) to 233 determine how many species can coexist based on this model. This approach attempts to find the 234 trait values for invaders that allow invasion in a given context. In this case, at each step, I find 235 the values for the trait $p_{i}$ that allows an invader to have a positive meta-community growth rate 236 (often written as $S\left(p_{i}\right)$ in the adaptive dynamics literature) in an assemblage of $n$ resident species,

$$
S_{\left\{p_{r n}\right\}}\left(p_{i}\right)>0
$$

237 i.e., . If this species can 1) coexist with the current resident strategies and resist exclusion by similar strategies (i.e. similar values $p$ ), then it is added the list of residents. Species are added one at a time until there is no value of $p_{i}$ that leads to positive metacommunity-scale growth rate. Because the approximated expression for growth-density covariance from eqn. 9 assumes small variation in supply rate and has singularities at $p_{i}=1$ and $p_{r}=1$, I use simulation of

242 eqn 1 to calculate invasion growth rate. Numerical simulations of the residents were run until 243 they reached a steady state. The resulting value of resource concentration was plugged into the 
244 matrix that described each patches' contribution to an invader's low-density growth-rate in patch

$245 x$. The dominant eigenvalue of this matrix is an estimate of the meta-community scale

246 low-density growth rate of the invader. For the simulations, I assumed Log-normal distributed

247 supply rates and trade-offs between and $p_{j}$ of the form where $Z$ is an arbitrary

$$
R_{j}^{*} \text { and } p_{j} \text { of the form } R_{j}^{*}-p_{j}^{\tau}=Z \text { where } Z \text { is an arbitrary }
$$

$\tau$

248 constant and affects the shape of the trade-off. The following example considers a linear

249 tradeoff, between competitive ability and dispersal i.e., ${ }^{\tau=1}$.

250 Figure 2 shows a contour plot for the invasibility of strategy $p_{i}$ in the presence of resident $p_{r}$. The

251 black region of Figure 2 represent negative invader growth rates (i.e. the invasion is

252 unsuccessful), the white regions shows areas of positive growth rates. It shows that any resident

253 strategy $p_{r}<1$ can be invaded by $p_{i}=1$. However, the strategy $p_{r}=1$ is also able to be invaded

$$
p_{i}<1
$$

254 by any . Thus, I set $p_{r 1}=1$ and looked for values of $p_{r 2}$ that could coexist with $p_{r 1}$ and were

255 not excluded by other similar strategies. Figure 3 shows that given $p_{r 1}=1$, any resident strategy

$256 p_{r 2}>0$ can be invaded by $p_{i}<p_{r 2}$. Thus, since $p_{r 2}=0$ can also coexist with $p_{r 1}=1$, it was added to the

257 resident list. Figure 3 also suggests that there is no third strategy $p_{i}$ that can coexist with $p_{r 1}=1$

$$
S_{\left\{p_{r 1=1}, p_{r 2=0}\right\}}\left(p_{i}\right)<0
$$

258 and $p_{r 2}=0$, since for all possible values of $p_{i}$ (i.e. a vertical line drawn from

$259 p_{r 2}=0$ passes only through the black region of the graph). This suggests that in this model, a 
$$
R_{j}^{*}
$$

260 tradeoff between competitive ability and dispersal $p_{j}$, allows coexistence of only two species.

261 To assure these results were robust, I simulated systems of simultaneous competition among

262 many species from along the trade-off manifold (Supplemental Appendix S2). Those

263 simulations also show an observed maximum of two coexisting species for nonlinear tradeoffs

$$
\tau<1
$$

264 with ; additional loss terms in the resource equation, and saturating growth responses. For

265 non-linear tradeoffs with ${ }^{\tau>1}$ no coexistence was possible.
\end{abstract}

Discussion

This analysis shows that spatial variation in resources allows for fewer coexistence mechanisms and lower potential species diversity compared to spatial variation in non-resource factors. Although spatial resource variation promotes coexistence if there is a tradeoff between competitive ability and the ability to retain offspring in good patches, this tradeoff allows the coexistence of only a 2 species.

273 effect, a powerful mechanism that allows the coexistence of many species. The storage effect is 274 absent because all species grow best in the same patches (high resource patches). As a result, 275 environmental responses and competition are perfectly and equally correlated for all species, 276 allowing no advantages in good patches when a species is rare. In other words, the feedback that 277 organisms have on the resource, make it impossible for any to specialize on a particular supply 278 rate along a gradient. 
Although the mechanism of resource competition analyzed in this model is simplified,

280 the qualitative results are quite general. For example, the relationship of $R^{*}$ to the

281 metacommunity-scale coexistence mechanisms described by scale transition theory does not

282 depend on the simplified model of local resource competition presented in eqns 1 . If the

283 establishment rate of seeds is a saturating function (e.g. Monod functions with equal half

284 saturation constants) of the amount of resources present, then ${ }^{p^{\prime}}$ is still a comparison of the (more 285 complicated) $R^{*}$ s of the invader and resident, and the relative importance of the variation 286 dependent mechanism is the same. The situation is more complex if species growth rates are 287 different non-linear functions of resource concentration, but, even in this case, spatial variation in 288 one only facilitate 2 species coexistence (Supplemental Appendix S2). In this model, competition was for a single resource. Increasing the number of limiting resources can increase the number of species that can coexist at equilibrium. Golubski et al.

291 (2008) found that a maximum of four species could coexist in a system with two resources, 292 heterogeneous supply rates and species that were capable of integrating growth across patches.

293 However, this was only possible with precise arrangement of species trait parameters, and if 294 resource supply rates of the resources were strongly negatively correlated across patches. I 295 presume that, in this case, adding resource factors does not increase the number of mechanisms; 296 the existing mechanism, growth-density covariance, simple works independently for each 297 resource; although this claim should be explored with further analysis. This analysis suggests that spatial resource heterogeneity is not capable of supporting the 299 robust stable coexistence of many plants species that is observed in many natural systems. The 300 reason for this is that the feedback species have on resources concentrations prevents 
301 specialization of different species at different supply rates. It is this kind of specialization of

302 different species along different points of a "niche" axis, which is measured by the spatial

303 storage effect, that allows the robust coexistence of many species. The results of this analysis are

304 consistent with the patterns found in the empirical literature, which finds much more support for

305 the relationship between species diversity and nonresource spatial heterogeneity than resource

306 heterogeneity. Taken together, this work suggests that the consideration whether a factor is a

307 resource or not is crucial for those attempting to understand real patterns in species diversity or

308 those interested in managing a habitat for increased species diversity.

Acknowledgements

310 I would like to thank Peter Chesson, Kay Gross, and Gary Mittelbach for helping improve this

311 manuscript. This is contribution No. TBD from the W.K. Kellogg Biological Station, Michigan

312 State University.

References

315 Abrams, P.A. 1988. Resource productivity - consumer species diversity: simple models of 316 competition in spatially heterogeneous environments. Ecology 69: 1418-1433.

317 Abrams, P.A., W.G. Wilson. 2004. Coexistence in metacommunities due to spatial variation in 318 resource growth rates; does R* predict the outcome of competition? Ecology Letters 7:929-940.

319 Amarasekare, P. 2003. Competitive coexistence in spatially structured environments: a synthesis. 320 Ecology Letters 6: 1109-1122.

321 Bakker, C., Blair, J.M., Knapp, A.M. 2003. Does resource availability, resource heterogeneity or 322 species turnover mediate changes in plant species richness in grazed grasslands? Oecologia 137: 323 385-391.

324 Chesson, P. 1994. Multispecies competition in variable environments. Theoretical Population 325 Biology 45: 227-276.

326 Chesson, P. 2000a. General theory of competitive coexistence in spatially-varying environments. 327 Theoretical Population Biology 58: 211-237. 
Chesson, P. 2000b. Mechanisms of maintenance of species diversity. Annual Review of Ecology, Evolution \& Systematics 31: 343-66.

Chesson, P. 2008. Quantifying and testing species coexistence mechanisms.-- In: F. Valladares, A. Camacho, A. Elosegui, C. Gracia, M. Estrada, J. C. Senar, and J. M. Gili, eds. Unity in Diversity: Reflections on Ecology after the Legacy of Ramon Margalef. Fundacion BBVA, Bilbao. pp. 119-164

S. A. H. Geritz, E. Kisdi, G. Meszena, J. A. J. Metz. 1998., Evolutionarily singular strategies and the adaptive growth and branching of the evolutionary tree, Evolutionary Ecology, 12:, 35-57.

Golubski, A.J., Gross, K.L., Mittelbach, G.G. 2008. Competition among plant species that interact with their environment at different spatial scales. Proceeding of Royal Society, $B$ 275:1897-906.

Lundholm, J.T. 2009. Plant Species diversity and environmental heterogeneity: spatial scale and competing hypothesis. Journal of Vegetation Science 20: 377-391.

Miller, A. D., Chesson, P. 2009. Coexistence in disturbance prone communities: how a resistance-resilience trade off generates coexistence via the storage effect. The American Naturalist 173, E30-E43.

Reynolds, H.R., Hungate, B.A., Chapin III, F.S., D’Antonio, C. 1997. Soil heterogeneity and plant competition in an annual grassland. Ecology 78: 2076-2090.

Reynolds, H.R., Mittelbach, G.G., Darcy-Hall, T., Houseman, G.R., Gross, K.L. 2007. No effect of varying soil resource heterogeneity on plant species richness in a low fertility grassland. Journal of Ecology, 95: 723-733.

Sears, A.L.W, Chesson, P. 2007. New methods for quantifying the spatial storage effect: an illustration with desert annuals. Ecology 88: 2240-2247.

Stevens, M.H.H., Carson, W. 2002. Resource quantity, not resource heterogeneity maintains plant diversity. Ecology Letters: 5: 420-426.

Tilman, D. 1982. Resource Competition and Community Structure. Princeton University Press.

Tilman, D., Pacala,S. 1993. The maintenance of species richness in plant communities. - In: Ricklefs, R. and Schluter, D., eds. Species Diversity in Ecological Communities, University of Chicago Press, Chicago. pp. 13-25. 